\title{
ACOMPAÑAMIENTO PSICOSOCIAL EN CUIDADORES PRIMARIOS INFORMALES: MINDFULNESS, CALIDAD DE VIDA Y APOYO SOCIAL A DISTANCIA
}

\author{
Luis Emilio Vicente Ramírez, Viridiana Medina Reyna, David Alberto Rodríguez Medina, Mario \\ Carranza Aguilar, José Joel Vázquez Ortega y Jesús Omar Manjarrez Ibarra \\ Universidad Autónoma Metropolitana, Unidad Iztapalapa \\ México
}

\begin{abstract}
RESUMEN
Los cuidadores primarios informales son un grupo de personas vulnerables de estrés crónico con escasa atención psicológica institucional. La pandemia por Covid-19 ha orillado a proporcionar intervención psicosocial a distancia (apoyo psicológico telefónico y videollamadas). En esta investigación se exploraron los efectos del acompañamiento psicosocial basado en mindfulness, el intercambio de experiencias psicosociales respecto al apoyo social, la percepción de la salud y la calidad de vida en cuidadores primarios informales de personas con enfermedades crónicas no transmisibles de la alcaldía Iztapalapa de la Ciudad de México. Mediante un diseño pre-post de un programa de intervención psicosocial, con una duración de ocho sesiones semanales, se obtuvieron medidas psicométricas de ansiedad, depresión, apoyo social, calidad de vida y percepción de salud. También se registró la tasa respiratoria y la temperatura periférica de los participantes. Los resultados mostraron una asociación significativa inversa entre el apoyo social afectivo y el nivel de ansiedad ( $r$ o $=0.97, p=0.006$ ), una correlación directa entre las relaciones sociales positivas y la salud emocional ( $r h o=-1, p<0.001$ ), una relación inversa entre la calidad de vida individual y la calidad de vida como cuidador primario ( $r$ ho $=-0.83, p=0.009$ ), una relación positiva entre la calidad de vida global y la salud física ( $r$ ho $=0.89, p=0.002$ ), una mejoría en el apoyo social instrumental ( $r h o=0.643, p=0.042$ ), además de una reducción somática (tasa respiratoria) en la mayoría de los ejercicios de mindfulness. Los resultados son parcialmente consistentes con los reportados en estudios antecedentes de este tipo de intervenciones en cuidadores primarios informales, y se propone diseñar, o en su caso adaptar, instrumentos de medición en su modalidad a distancia.
\end{abstract}

Palabras Clave:

tasa respiratoria, temperatura periférica, intervención psicosocial a distancia, autocuidado, asertividad y mindfulness.

\section{PSYCHOSOCIAL ACCOMPANIMENT IN INFORMAL PRIMARY CAREGIVERS: MINDFULNESS, QUALITY OF LIFE AND DISTANCE SOCIAL SUPPORT}

\begin{abstract}
Informal primary caregivers are a group of people vulnerable to chronic stress with little institutional psychological care. The Covid-19 pandemic has led to remote psychosocial intervention (telephone psychological support and video calls). In this research, the effects of psychosocial support based on mindfulness, the exchange of psychosocial experiences on social support, the perception of health, and the quality of life in informal primary caregivers of people with chronic non-communicable diseases of the Iztapalapa municipality of the CDMX. Through a pre-post design of a psychosocial intervention program, with a duration of eight weekly sessions, psychometric measures of anxiety, depression, social support, quality of life, and perception of health were obtained. The respiratory rate and peripheral temperature of the participants were also recorded. The results showed a significant inverse association between affective social support and anxiety level (rho $=0.97, p=0.006$ ), a direct correlation between positive social relationships and emotional health ( $r h o=-1, p<0.001$ ), an inverse relationship between individual quality of life and quality of life as a primary caregiver ( $r h o=-0.83, p=0.009$ ), a positive relationship between global quality of life and physical health (rho $=0.89, p=0.002$ ), an improvement in instrumental social support (rho $=0.643, p=0.042$ ), in addition to a somatic reduction (respiratory rate) in most mindfulness exercises. The results are partially consistent with those reported in antecedent studies on this type of intervention in informal primary caregivers and it is proposed to design or, where appropriate, adapt measurement instruments in their remote modality..
\end{abstract}

Keywords:

respiratory rate, peripheral temperature, remote psychosocial intervention, self-care, assertiveness and mindfulness.

Bitácora del Artículo:

| Recibido: 11 de Agosto de 2021 | Aceptado: 04 de Enero de 2022 | Publicado en línea: Enero - Junio de 2021 | 
Artículo Empírico | Acompañamiento psicosocial en cuidadores primarios... | Vicente-Ramírez, et al.

\title{
Autoría y Derechos de Propiedad Intelectual
}

\author{
ACOMPAÑAMIENTO PSICOSOCIAL EN CUIDADORES PRIMARIOS \\ INFORMALES: MINDFULNESS, CALIDAD DE VIDA Y APOYO SOCIAL A \\ DISTANCIA
}

\begin{abstract}
Luis Emilio Vicente Ramírez, Viridiana Medina Reyna, David Alberto Rodríguez Medina, Mario
Carranza Aguilar, José Joel Vázquez Ortega y Jesús Omar Manjarrez Ibarra

Universidad Autónoma Metropolitana, Unidad Iztapalapa

México
\end{abstract}

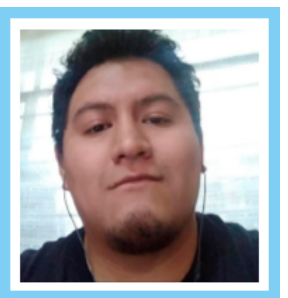

Luis Emilio Vicente Ramírez

UAM Iztapalapa

Correo: zerovicente@hotmail.com

Egresado de la licenciatura en Psicología Social, ponente en el encuentro estudiantil "Intercambio académico de experiencias comunitarias de psicología social psicológica".

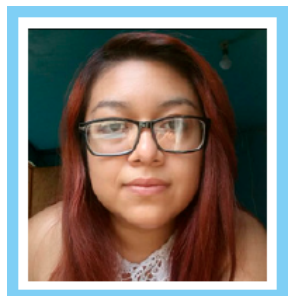

Viridiana Medina Reyna

UAM Iztapalapa

Correo: medina_viri@hotmail.com

Egresada de la licenciatura en Psicología Social, ponente en el encuentro estudiantil "Intercambio académico de experiencias comunitarias de psicología social psicológica"

Autor de Correspondencia: David Rodríguez drodriguezm@xanum.uam.mx

\section{CONTRIBUCIÓN DE lOS AUtORES}

Luis Emilio Vicente Ramírez es responsable del trabajo en campo, recolección y análisis de datos y redacción del manuscrito | Viridiana Medina Reyna estuvo a cargo del trabajo en campo, recolección y análisis de datos y redacción del manuscrito | David Alberto Rodríguez Medina participó en la revisión del manuscrito y análisis de datos | Mario Carranza Aguilar colaboró en el trabajo de campo. | José Joel Vázquez Ortega participó en la Revisión del manuscrito | Jesús Omar Manjarrez Ibarra ayudó con la redacción del manuscrito.

\section{AGRADECIMIENTOS}

Apreciamos el apoyo del grupo de cuidadores primarios "Guerreras de Samarehi" y a la organización de colonos de San Miguel Teotongo. Agradecemos también al Área de Procesos Psicosociales y de los Fenómenos Colectivos de la UAM-Iztapalapa, así como al Dr. Javier Rodríguez Lagunas, Jefe del Departamento de Sociología y al Mtro. José Manuel Gutiérrez Fiall, Coordinador de la Licenciatura en Psicología Social.

\section{DATOS dE FiLIACIÓN DE los Autores}

Universidad Autónoma Metropolitana Iztapalapa

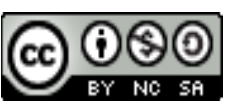

Copyright: (c) 2022 Vicente-Ramírez, L. E.; Medina-Reyna, V.; Rodríguez-Medina, D.A.; Carranza-Aguilar, M.; Vázquez-Ortega, J.J. \& Manjarrez-lbarra, J.O. Este es un artículo de acceso abierto distribuido bajo los términos de la licencia Creative Commons Reconocimiento-NoComercial 4.0 Internacional, por lo que su contenido gráfico y escrito se puede compartir, copiar y redistribuir total o parcialmente sin necesidad de permiso expreso de sus autoras con la única condición de que no se puede usar con fines directamente comerciales y los términos legales de cualquier trabajo derivado deben ser los mismos que se expresan en la presente declaración. La única condición es que se cite la fuente con referencia a la Revista Digital Internacional de Psicología y Ciencia Social y a sus autoras. 


\section{TABLA DE CONTENIDO}

MÉTODO

Diseño de investigación, 5

Participantes, 5

Materiales, 5

Procedimiento, 5

Instrumentos, 6

Análisis estadísticos, 7

RESULTADOS

DiscusióN

CONCLUSIONES

REFERENCIAS 
a Organización Mundial de la Salud (OMS) define la calidad de vida como "las percepciones individuales de una persona, de su posición en la vida en el contexto de su cultura y del sistema de valores en que vive y en relación con sus objetivos, expectativas, normas y preocupaciones" (OMS, 1998). Un cuidador primario (CP) es aquel que asume responsabilidad del paciente ayudándole a efectuar todas las actividades que éste no puede hacer por sí mismo; además cumple una función de apoyo afectivo. Por lo común, es un miembro de la red social inmediata del paciente (un familiar, un amigo o incluso un vecino), y regularmente no percibe un apoyo económico ni tiene capacitación previa a sus funciones de cuidador. Los CP por lo general son mujeres amas de casa sin estudios y con un parentesco directo con el paciente (Islas et. al., 2006). Los CP pueden ignorar los efectos biopsicosociales de la sobrecarga y sus implicaciones en su salud (física y emocional). El CP incrementa de manera progresiva el tiempo de cuidado de su familiar y disminuye el tiempo de descanso, puede desarrollar una conducta de sobreprotección, lo que conlleva mayor tiempo de trabajo y dedicación, lo cual puede generar estrés y/o agotamiento (Camacho, Yokebed y Jiménez, 2010). Debido a la labor que desempeñan cubriendo las necesidades básicas, sociales y emocionales de sus dependientes, los cuidadores primarios pueden quedar sometidos a una sobrecarga de trabajo (Cardona et al., 2013). Los efectos de padecer una enfermedad crónica y/o degenerativa no sólo afectan a la persona que la padece sino al resto de su entorno, y sobre todo a quien desempeña la labor de cuidador primario, que por lo común es algún familiar. Es así como los efectos de la sobrecarga del trabajo pueden afectar de manera física y mental, ocasionando depresión, ansiedad, aislamiento y fatiga, entre otras (Hernández-Cantú et al., 2017).

El apoyo social se refiere a las redes sociales de las que dispone una persona y a los recursos emocionales, instrumentales y materiales que pueden ofrecerle, lo cual influye en la calidad de vida. Puerto-Pedraza y Carrillo-González (2015) encontraron que el apoyo social en cuidadores influye en el proceso de satisfacción del cuidador, proporciona apoyo en situaciones de complejidad y contribuye de modo positivo en su bienestar físico, psicológico, social y espiritual. Por tanto, formar y fortalecer sus redes de apoyo social tiene una función protectora y amortiguadora. La convivencia es parte esencial del ser humano, por lo que el tipo de relación que tenga con los demás influye en la calidad de apoyo social otorgado y percibido, tanto de las redes sociales como en los familiares. Una convivencia se refiere no sólo a compartir el espacio físico, sino también al reconocimiento de la diversidad de los miembros que forman parte de estos espacios, ya que comparten un sistema de convenciones y normas, por lo que es importante que cada persona considere sus propios intereses y comprenda los de los demás para favorecer el bien común, y a su vez facilitar la resolución de conflictos (Ortega-Ruiz, 2006), fortaleciendo la capacidad de identificarse con los otros y comprender sus puntos de vista mediante las experiencias sociales que se comparten; a su vez, éstas fomentan un sentido de pertenencia común (Bayón y Saraví, 2019) y es una fuente de red de apoyo social funcional.

La red de apoyo social puede fomentar las prácticas de autocuidado y la calidad de vida que influye tanto de manera personal como social, generando promoción y prevención de la salud, las cuales dotan a las personas de recursos que les permitan decidir y discernir de manera responsable e informada el efecto de sus propias conductas sobre su salud, fomentando conductas de protección o evitando las de riesgo, en relación con el cuidado de sí mismo (Camacho-Gutiérrez et al., 2014).

Se han probado diversas intervenciones para disminuir los efectos de sobrecarga y estrés en el cuidador, entre ellas las basadas en mindfulness. Diversos estudios han mostrado mejorías en el bienestar psicológico de quienes practican mindfulness en una revisión sistemática en cuidadores de pacientes con discapacidad mental, y se encontró que esta práctica tiene efectos positivos a largo plazo sobre la depresión, manteniendo niveles aceptables de estrés, ansiedad y sobrecarga (Grandi et al., 2018).

El mindfulness se define por ser una práctica que consiste en "prestar atención de manera intencional al momento presente, sin juzgar" (Kabat-Zinn, 1990); proviene de una práctica de meditación budista que busca focalizar la atención en tiempo presente, de manera deliberada sin emitir juicios de valor con el propósito de cambiar las relaciones entre los pensamientos, los sentimientos y las sensaciones físicas. Se han encontrado múltiples beneficios en la práctica de mindfulness, como bienestar psicológico, disminución de dolor crónico, alivio de síntomas de ansiedad y depresión, entre otros (Ruiz-Fernandez et al., 2012).

El apoyo social percibido y las técnicas basadas en mindfulness buscan un nivel de activación fisiológico predominantemente parasimpático, que inhibe las funciones defensivas tanto físicas como conductuales, propiciando una reducción en la tasa cardiaca y respiratoria, así como un mejor flujo sanguíneo reflejado con el incremento de la temperatura periférica, los cuales regulan la actividad inflamatoria y el estrés crónico (Rodríguez-Medina et al., 2018). 
El objetivo del este estudio es explorar el efecto de las prácticas de promoción y prevención de la salud y estrategias de mindfulness sobre la tasa respiratoria, la temperatura periférica y factores psicosociales asociados a la calidad de vida en personas cuidadoras de enfermedades crónicas no transmisibles.

\section{MÉTodo}

\section{Diseño de investigación}

Para explorar los efectos de la intervención sobre los participantes se utilizaron dos diseños de investigación (Hernándes-Sampieri, Fernández-Collado y Baptista-Lucio, 2014). El primero fue un diseño exploratorio prepost intervención para las medidas de evaluación psicométrica. El segundo diseño, exploratorio, de medidas repetidas, de un solo grupo para las medidas psicofisiológicas.

\section{Participantes}

Muestra no probabilística; por conveniencia se consideraron participantes que fueran cuidadores primarios de dependientes con diversos padecimientos crónicos y/o degenerativos, que fueran residentes de la colonia San Miguel Teotongo, Iztapalapa, Ciudad de México, y forman parte del Sistema Público de Cuidados. La edad media fue de 52.5 años $(\mathrm{DE}=6.32)$ con un nivel de escolaridad entre primaria y carrera técnica. Los cuidadores primarios suelen ser sobre todo mujeres familiares de dependiente, ya que culturalmente se asocia el cuidado a las mujeres; en el grupo que se formó, solo participó un hombre; sin embargo, debido a las dificultades de conexión y tecnológicas, no logró participar, quedando el grupo conformado sólo por mujeres.

\section{Materiales}

Los participantes utilizaron dispositivos móviles y/o equipos de cómputo para efectuar las videoconferencias, para las cuales se usó la plataforma "Google meet".

Para esta investigación se utilizaron las estrategias de mindfulness sugeridas por la Brigada de Salud Mental del Instituto Nacional de Ciencias Médicas y Nutrición Salvador Zubirán (INCMNSZ, 2020).

\section{Procedimiento}

El grupo se conformó por cuidadores primarios de San miguel Teotongo que tuvieron asesoría psicológica por medio de llamadas telefónicas. Las mediciones pre y postest (recolectadas en septiembre de 2020 y mayo de 2021) se hicieron con los instrumentos psicométricos in- dividualmente previo y posterior a las sesiones grupales con una duración de 1.5 horas, cada semana (tabla 1 ).

Tabla 1.

Organización de las sesiones grupales.

\begin{tabular}{|c|c|c|c|c|}
\hline SESIÓN & NOMBRE & OBJETIVO & DESCRIPCIÓN & TÉCNICA \\
\hline 1 & $\begin{array}{l}\text { Aprender a } \\
\text { no agredir al } \\
\text { otro. }\end{array}$ & $\begin{array}{l}\text { Fomentar la } \\
\text { comunicación } \\
\text { y la sana } \\
\text { convivencia } \\
\text { sin agresión. }\end{array}$ & $\begin{array}{l}\text { Por medio de } \\
\text { presentaciones } \\
\text { PowerPoint y } \\
\text { cortometrajes. }\end{array}$ & $\begin{array}{l}\text { Respirar con } \\
\text { atención } \\
\text { plena. }\end{array}$ \\
\hline 2 & $\begin{array}{l}\text { Aprender a } \\
\text { comunicarse. }\end{array}$ & $\begin{array}{l}\text { Fomentar la } \\
\text { comunicación } \\
\text { asertiva. }\end{array}$ & $\begin{array}{l}\text { Por medio de } \\
\text { una presentación } \\
\text { PowerPoint } \\
\text { y la actividad } \\
\text { "malentendido". }\end{array}$ & $\begin{array}{l}\text { Escaneo } \\
\text { corporal. }\end{array}$ \\
\hline 3 & $\begin{array}{l}\text { Aprender a } \\
\text { interactuar. }\end{array}$ & $\begin{array}{l}\text { Fomentar la } \\
\text { interacción e } \\
\text { integración } \\
\text { grupal. }\end{array}$ & $\begin{array}{l}\text { Por medio de } \\
\text { una presentación } \\
\text { PowerPoint y la } \\
\text { actividad "sigue el } \\
\text { cuento". }\end{array}$ & $\begin{array}{l}\text { Siendo } \\
\text { consciente } \\
\text { adentro y } \\
\text { afuera. }\end{array}$ \\
\hline 4 & $\begin{array}{l}\text { Aprender a } \\
\text { decidir en } \\
\text { grupo. }\end{array}$ & $\begin{array}{l}\text { Analizar el } \\
\text { manejo de } \\
\text { crisis y } \\
\text { negociación } \\
\text { personal e } \\
\text { interpersonal } \\
\text { en una } \\
\text { situación de } \\
\text { conflicto. }\end{array}$ & $\begin{array}{l}\text { Por medio de } \\
\text { una presentación } \\
\text { PowerPoint y } \\
\text { la actividad de } \\
\text { elaborar un escudo, } \\
\text { lema y nombre } \\
\text { propio del grupo. }\end{array}$ & $\begin{array}{l}\text { Mindfulness } \\
\text { en tu mano. }\end{array}$ \\
\hline 5 & $\begin{array}{l}\text { Aprender a } \\
\text { cuidarse. }\end{array}$ & $\begin{array}{l}\text { Informar a los } \\
\text { participantes } \\
\text { sobre la } \\
\text { importancia } \\
\text { del } \\
\text { autocuidado y } \\
\text { cómo llevarlo } \\
\text { a cabo. }\end{array}$ & $\begin{array}{l}\text { Por medio de } \\
\text { una presentación } \\
\text { PowerPoint } \\
\text { y cápsulas } \\
\text { informativas sobre } \\
\text { alimentación y } \\
\text { actividad física, } \\
\text { llevadas a cabo cada } \\
\text { semana. }\end{array}$ & $\begin{array}{l}\text { Observación } \\
\text { de la } \\
\text { respiración. }\end{array}$ \\
\hline 6 & $\begin{array}{l}\text { Aprender } \\
\text { a cuidar el } \\
\text { entorno } \\
\text { social. }\end{array}$ & $\begin{array}{l}\text { Informar sobre } \\
\text { la importancia } \\
\text { de cuidar } \\
\text { el entorno } \\
\text { social y a su } \\
\text { vez el medio } \\
\text { ambiente. }\end{array}$ & $\begin{array}{l}\text { Por medio de } \\
\text { una presentación } \\
\text { PowerPoint y la } \\
\text { actividad "Aprender } \\
\text { a cuidar el entorno". }\end{array}$ & $\begin{array}{l}\text { Acepta la } \\
\text { ansiedad. }\end{array}$ \\
\hline 7 & $\begin{array}{l}\text { Aprender } \\
\text { a cuidar el } \\
\text { saber social. }\end{array}$ & $\begin{array}{l}\text { Informar a los } \\
\text { participantes } \\
\text { sobre la } \\
\text { importancia } \\
\text { del saber } \\
\text { común, } \\
\text { tradiciones y } \\
\text { costumbres. }\end{array}$ & $\begin{array}{l}\text { Por medio de } \\
\text { una presentación } \\
\text { PowerPoint y la } \\
\text { actividad "Valorar } \\
\text { el saber cultural y } \\
\text { educativo". }\end{array}$ & $\begin{array}{l}\text { Observación } \\
\text { de la } \\
\text { respiración. }\end{array}$ \\
\hline 8 & $\begin{array}{l}\text { Organización } \\
\text { del } \\
\text { encuentro } \\
\text { final. }\end{array}$ & $\begin{array}{l}\text { Participación } \\
\text { activa del } \\
\text { grupo para la } \\
\text { última sesión. }\end{array}$ & $\begin{array}{l}\text { Utilizando los } \\
\text { principios de } \\
\text { convivencia, planear } \\
\text { la organización } \\
\text { y actividades a } \\
\text { realizar.. }\end{array}$ & $\begin{array}{l}\text { Respirar con } \\
\text { atención } \\
\text { plena. }\end{array}$ \\
\hline
\end{tabular}


Las sesiones grupales estuvieron diseñadas mediante segmentos de participación; los facilitadores moderaban espacios para una bienvenida, un segmento para fomentar/instruir ejercicios físicos y cápsulas informativas de alimentación adecuadas para la media de edad de los participantes, una breve información acerca de "los principios básicos de convivencia" y una actividad grupal para reforzar la información y una técnica de mindfulness con sus respectivos momentos de medición; debido a las circunstancias de las sesiones, muchas veces los participantes estaban acompañados por amigos y familiares que los asistían en el uso de la tecnología implicada.

Durante las ocho sesiones se tomó una medición fisiológica antes y después de la práctica de las técnicas en "mindfulness". En las primeras dos sesiones se registraron mediciones basadas en temperatura periférica, pero debido a que se desconoce la variabilidad de la temperatura ambiente de las circunstancias ecológicas de cada participante, y que en diversos manuales se indica debe ser la misma, se optó por cambiar la medición por la tasa respiratoria por minuto, por lo que las restantes se tomaron mediante frecuencia respiratoria.

\section{Instrumentos}

Patient Health Questionnaire (PHQ-4) (Löwe et al., 2010). Cuestionario de tamizaje para valorar presencia de ansiedad y depresión. Tiene una varianza explicada de $84 \%$ y una confiabilidad de 0.82 a 0.90 . El PHQ está basado con los criterios del DSM-IV, traducido al español por Diez-Quevedo, Rangil, Sanchez-Planell, Kroenke y Spitzer (2001), con una población de 1,003 pacientes españoles y está validada para población general. La ansiedad y la depresión son esquemas derivados de cuadros de estrés crónico; en este sentido se espera que ambas variables estén presentes. En población mexicana ha sido utilizado en diversas investigaciones (Rodríguez-Medina et al., 2018; Rodríguez, Vázquez Ortega y Alonso Hernández, 2020; Carranza et al., 2020; Rodríguez-Medina y Hernández Pozo, 2021).

Primary Care Posttraumatic Stress Disorder (PC-PTSD) (Prins et al., 2003). Instrumento de autorreporte de tamizaje para valorar la existencia de estrés postraumático. Es un cuestionario de cuatro ítems con respuestas dicotómicas. Tiene un nivel de confiabilidad test-retest de 0.83.

Cuestionario General de Salud SF-12 (Short Form 12 Health Curvey, SF-12) (Cisneros-Huerta, 2007). Instrumento de autorreporte de tamizaje sobre la salud general derivado del instrumento SF-36. Evalúa la capacidad funcional de las personas, definiendo un estado positivo y negativo de la salud física y mental por medio de ocho subescalas: 1) función física; 2) rol físico; 3) dolor corporal; 4) salud mental; 5) salud general; 6) vitalidad; 7) función social, y 8) rol emocional. El SF-12 presenta una validez convergente con el instrumento original SF-36, de $r=0.737(p<0.01)$ para la dimensión física, y de $r=0.714(p<0.01)$ para la dimensión mental en población mexicana.

Cuestionario de Apoyo Social de MOS (AS-MOS) (Sherbourne y Stewart, 1991). Instrumento autoaplicable para la evaluación del apoyo social, breve; tiene cuatro dimensiones: 1) afectiva (demostración de amor, cariño y empatía); 2) interacción social positiva (posibilidad de contar con personas para comunicarse); 3) instrumental (posibilidad de ayuda doméstica), y 4) informacional (posibilidad de asesoramiento, consejo e información). El Alpha de Cronbach de las subescalas está entre 0.93 y 0.96.

Cuestionario de Calidad de Vida de la OMS (WHOQOL-BREF) (OMS, 1996). Adaptación a la lengua española, conformado por 26 ítems, cada uno con cinco opciones de respuesta, utilizado para medir calidad de vida, sin importar las diferencias culturales, agrupadas en las cinco dimensiones propuestas por la OMS: 1) percepción global (dos ítems); 2) salud física (siete ítems); 3) salud psicológica (seis ítems); 4) ambiente (ocho ítems), y 5) relaciones sociales (tres ítems). Para la aplicación del instrumento se utilizó la validación mexicana, donde se excluyeron siete ítems para un mejor ajuste, dando como resultado en cada factor: 1$)$ percepción global (dos ítems); 2) salud física (cuatro ítems); 3) salud psicológica (cuatro ítems); 4) ambiente (seis ítems), y 5) relaciones sociales (tres ítems), con un $\alpha \geq 0.70$ para todas las dimensiones y un $\alpha=0.895$ global (López-Huerta et al., 2017).

Escala de valoración de calidad de vida y satisfacción en familiares cuidadores (ECVS-FC). (Escala de valoración de calidad de vida y satisfacción en familiares cuidadores (ECVS-FC), propuesta por Grau, Chacón, Grau, García, Ojeda (2006; citado en Expósito et al., 2018). Cuestionario de autoinforme de 13 ítems, con respuesta dicotómica (sí/no), y en caso de respuesta afirmativa se proporciona una escala de cuatro puntos referido a la intensidad (bajo, medio, alto y muy alto), agrupado en dos dimensiones de calidad de vida (10 ítems) y satisfacción (tres ítems) (Expósito, Martín y Oviedo, 2019).

Tasa respiratoria (Berman y Snyder, 2013). La frecuencia respiratoria consiste en la cantidad de ciclos de inhalación y exhalación que realiza una persona por minuto, que es sensible a los cambios en el estado emocional como relajación y estrés. Se ha observado que la frecuencia respiratoria tiende a disminuir al inducirse un estado de relajación, y por el contrario, aumenta con estímulos estresantes o de alta activación. El rango de la tasa respiratoria por minuto de una persona adulta es de 12-20 ciclos por minuto. 
Temperatura periférica (Ioannou, Gallese y Merla, 2014). La temperatura periférica está relacionada con las reacciones emocionales porque el flujo sanguíneo cambia por la vasoconstricción. En las yemas de los dedos y las palmas de las manos, las temperaturas medias que se han registrado para humanos en estado de reposo son $32.3{ }^{\circ} \mathrm{C}$ para la palma de la mano y $30-34{ }^{\circ} \mathrm{C}$ para las yemas de los dedos, comprendiendo que a mayor temperatura periférica se presenta una mejor vasodilatación, permitiendo un mayor flujo sanguíneo, que se presenta en estados emocionales de seguridad y relajación; en contraparte, un descenso de temperatura en los dedos de las manos significa una mayor actividad simpática debido al esfuerzo (cognitivo-afectivo).

Asistencia a las sesiones semanales

En cada sesión participaron de entre tres a siete participantes cuidadores informales. En la sesión 1 se presentaron $n=5$, en la sesión 2 participaron $n=6$, y en la sesión 3 la asistencia fue de $n=5$. En la sesión 4 se tuvo la participación de $n=4$, y en la sesión 5 fueron $n=3$; después, en la sesión 6 participaron $n=4$, en la sesión 7 fueron $n=5$, y en la sesión 8 la participación fue de $n=3$.

\section{Análisis estadísticos}

Se extrajeron los datos estadísticos de tendencia central y dispersión de la escala WHOQOL-BREF y de la escala ECVS-FC. Para las medidas fisiológicas se utilizó la prueba Wilcoxon y la prueba Friedman, así como para las escalas SF-12, AS-MOS, PHQ-4 y el PC-PTSD. Se utilizó la prueba de correlación rho de Spearman para el análisis de las puntuaciones psicométricas. Los análisis de comparación de dos muestras se hicieron en la calculadora en línea Al-Therapy Statistics. Las correlaciones se efectuaron en Past4, versión 0.3. Para todos los análisis se consideró un $\alpha=0.05$.

\section{Resultados}

\section{Temperatura periférica (sesiones 1 y 2)}

En la sesión 1 la temperatura periférica presentó un descenso entre la medición inicial y el momento previo a la técnica "Respirar con atención plena", el cual de manera grupal logró aumentar la temperatura sin diferencia estadísticamente significativa $(X 2=0.5,(2), \mathrm{p}>0.05)$.

\section{Tasa respiratoria (sesiones 3-8)}

En la sesión 3, la estrategia "Siendo consciente adentro y afuera", la tasa respiratoria no mostró cambios estadísticamente significativos entre ninguno de los tres momentos de medición (X2 = 0.5, (2), p > 0.05). Durante la se- sión 4 hubo una reducción significativa con el ejercicio de "Mindfulness en tu mano" (X2 = 0.5, (2), p = 0.05).

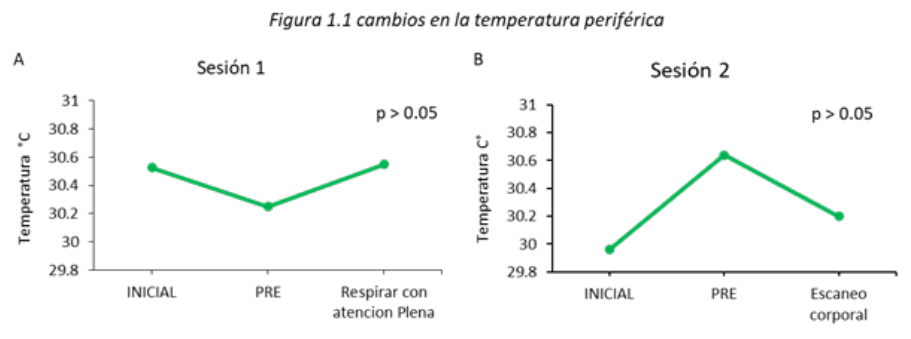

Figura 1.

Cambios en la temperatura periférica.

En la sesión 5 la tasa respiratoria no exhibió cambio estadísticamente significativo con la técnica "Observación de la respiración" (X2 = 0.5, (2), p > 0.05). Respecto a la sesión 6 , no hubo cambios en la tasa respiratoria con el ejercicio "Acepta la ansiedad" (X2 = 0.5, (2), p > 0.05). En la sesión 7 no se reportaron cambios con la técnica "Observación de la respiración" (X2 = 0.5, (2), p > 0.05). Durante la sesión 8 la tasa respiratoria no manifestó cambio estadísticamente significativo con la técnica "Respirar con atención plena" (X2 = 0.5, (2), p > 0.05).
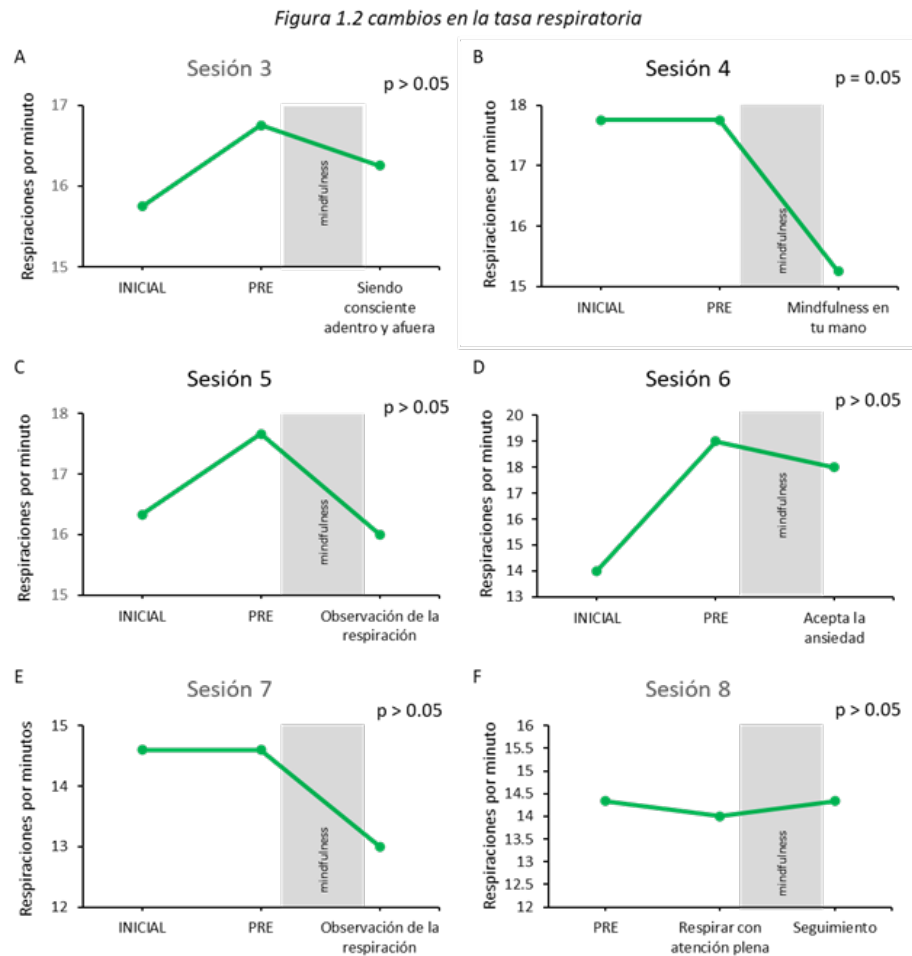

Figura 2.

Cambios en la tasa respiratoria.

Aun cuando no se encontraron cambios estadísticamente significativos en la mayoría de las sesiones de 
la tasa respiratoria, se muestra una tendencia a la baja después de cada técnica de mindfulness.

\section{Evaluaciones psicométricas}

Las evaluaciones psicométricas están compuestas por una comparación entre el inicio del programa de intervención y el término. En los niveles psicométricos se presentó un nivel amplio de variaciones sin tendencias significativas de cambio, con excepción del factor instrumental del AS-MOS, que mostró un incremento favorable estadísticamente significativo $(Z=2.032, \mathrm{p}=$ $0.042, r=0.643$ ) que fue de $M d=13$ (D.E. 4.494) a $\mathrm{Md}=16$ (D.E.= 5.568) en el nivel del instrumento relacionado con la percepción de apoyo doméstico; éste se omitió de la figura 3 porque sólo hubo distribución favorable. Dentro del PC-PTSD, únicamente una persona respondió "sí" a los cuatro ítems, lo cual implica un tamizaje afirmativo de padecer síndrome de estrés postraumático.

Tabla 2.

Diferencias de instrumentos psicométricos.

\begin{tabular}{|c|c|c|c|c|c|}
\hline INSTRUMENTO & MD PRE & $\begin{array}{l}\text { MD } \\
\text { Post }\end{array}$ & Z & $\mathbf{P}$ & R \\
\hline SF-12 Física & 16 & 15 & 0.184 & 0.854 & -0.058 \\
\hline $\begin{array}{l}\text { SF-12 } \\
\text { Emocional }\end{array}$ & 16 & 15 & 0 & 1 & 0 \\
\hline SF-12 Social & 5 & 3 & 0.552 & 0.581 & 0.175 \\
\hline $\begin{array}{l}\text { AS-MOS } \\
\text { Instrumental }\end{array}$ & 13 & 16 & 2.032 & $0.042^{*}$ & -0.643 \\
\hline $\begin{array}{l}\text { AS-MOS } \\
\text { Emocional }\end{array}$ & 32 & 40 & 0.730 & 0.465 & -0.231 \\
\hline $\begin{array}{l}\text { AS-MOS } \\
\text { Relaciones } \\
\text { Soc. }\end{array}$ & 15 & 19 & 0.412 & 0.680 & -0.130 \\
\hline $\begin{array}{l}\text { AS-MOS } \\
\text { Afectivo }\end{array}$ & 14 & 15 & 0.535 & 0.593 & -0.16 \\
\hline $\begin{array}{l}\text { PHQ-4 } \\
\text { Ansiedad }\end{array}$ & 1 & 0 & 1.105 & 0.269 & 0.349 \\
\hline $\begin{array}{l}\text { PHQ-4 } \\
\text { Depresión }\end{array}$ & 3 & 2 & 0.271 & 0.786 & 0.086 \\
\hline \multicolumn{6}{|c|}{$\begin{array}{l}\text { SF12 Física = Cuestionario de salud física, forma breve. SF12 Emocional = } \\
\text { Cuestionario de salud emocional, forma breve. SF12 Social = Cuestionario de } \\
\text { salud social, forma breve. AS-MOS Instrumental = Cuestionario de apoyo social } \\
\text { de MOS instrumental. AS-MOS Emocional = Cuestionario de apoyo social de } \\
\text { MOS emocional. AS-MOS Relaciones Soc. = Cuestionario de apoyo social de } \\
\text { MOS relaciones sociales. AS-MOS Afectivo = Cuestionario de apoyo social de } \\
\text { MOS afectivo. PHQ-4 Ansiedad = Cuestionario de salud del paciente ansiedad, } \\
\text { forma ultrabreve. PHQ-4 Depresión }=\text { Cuestionario de salud del paciente } \\
\text { depresión, forma ultrabreve. }{ }^{*}<0.05, r=\text { tamaño del efecto. }\end{array}$} \\
\hline
\end{tabular}

En la figura 3 se muestran las diferencias (pre-post) en distribuciones porcentuales por instrumento; sólo hubo cambios desfavorables en AS-MOS R.S. y en SF12 Emocional en esta distribución.

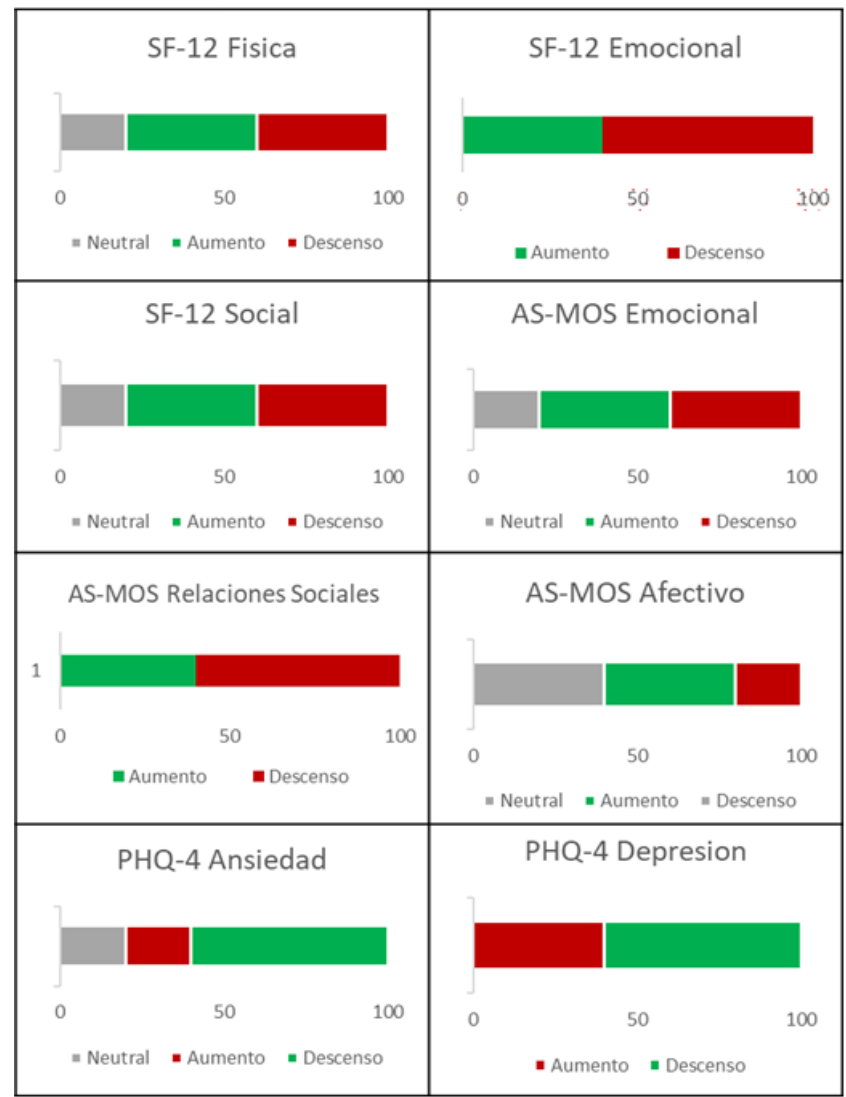

Figura 3.

Distribución porcentual por instrumento

Nota: Las distribuciones muestran la diferencia entre las respuestas del pre y el post de cada instrumento.

Los cambios favorables se muestran en verde, los desfavorables en rojo y los que se mantuvieron igual en gris. SF-12 Física = factor físico, SF-12 Emocional = factor emocional, SF-12 Social = factor social, AS-MOS Emocional = apoyo emocional, AS-MOS Relaciones sociales $=$ apoyo social, AS-MOS Afectivo = apoyo afectivo, PHQ-4 Ansiedad = posibilidad de ansiedad, PHQ-4 Depresión = posibilidad de depresión.

\section{Correlaciones de instrumentos psicométricos}

En la figura 4 los factores correspondientes a los instrumentos de calidad de vida reportan una correlación significativa ( $r$ o $=0.89, p=0.002$ ) entre la percepción global de calidad de vida y la salud física, ambos pertenecientes al instrumento de la OMS. También se encontró una correlación inversa entre la calidad de global y la percepción de calidad de vida del cuidador, ambos pertenecientes al instrumento CVC. 


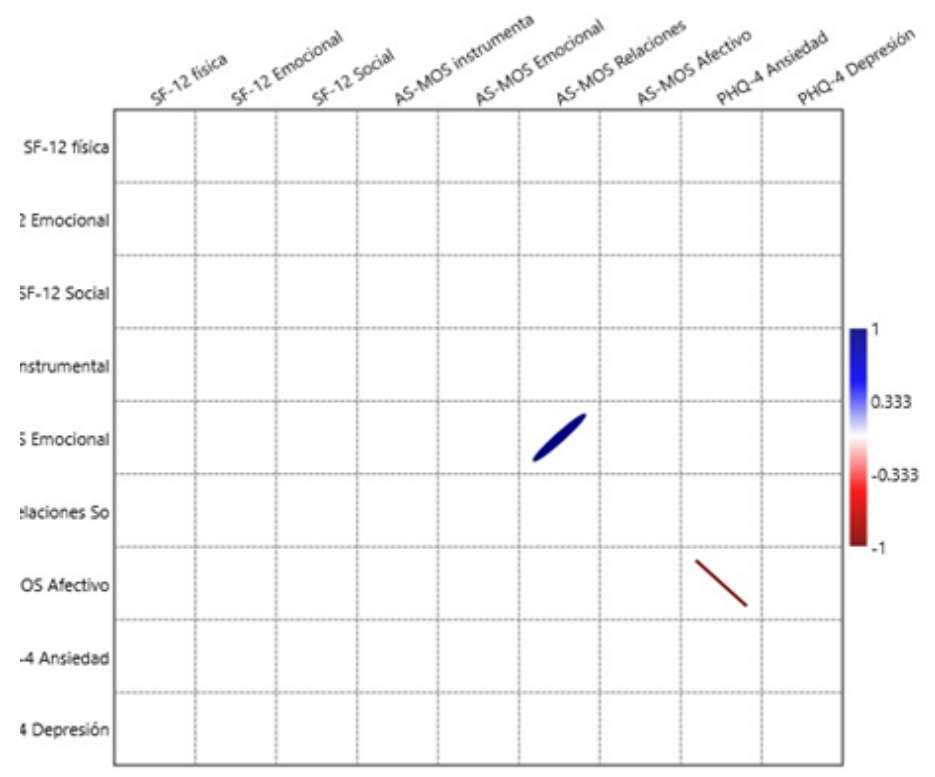

Figura 4.

Correlaciones entre los factores psicométricos.

Nota: Las correlaciones directamente proporcionales se presentan en azul, las inversamente proporcionales se encuentran en color rojo, las que están resaltadas tienen una diferencia estadísticamente significativa $(\alpha=0.05)$. SF-12 Física = factor físico, SF-12 Emocional = factor emocional, SF-12 Social = factor social, AS-MOS Emocional = apoyo emocional, AS-MOS Relaciones sociales $=$ apoyo social, AS-MOS Afectivo = apoyo afectivo, PHQ-4 Ansiedad = posibilidad de ansiedad, PHQ-4 Depresión = posibilidad de depresión.

En la figura 5 los factores correspondientes a los instrumentos psicométricos mostraron dos correlaciones estadísticamente significativas; el primero es una relación entre el factor emocional y el relacional pertenecientes al instrumento AS-MOS $(r=-1, p<0.001)$. La segunda relación es inversa y pertenece al factor afectivo del AS-MOS y el factor de Ansiedad perteneciente al PHQ-4 ( $r=0.97, p=0.006)$.

\section{Discusión}

Con base $\mathrm{s}$ los objetivos planteados se exploraron los efectos de la promoción de la salud, la práctica de ejercicios de mindfulness de la temperatura periférica y la tasa respiratoria, así como los componentes de la calidad de vida en personas cuidadoras. Los resultados indicaron que el ejercicio de la sesión 1, "respirar con atención plena", mostró una mejoría grupal de la temperatura periférica; este ejercicio es de fácil comprensión y de carácter visual; esto pudo facilitar el entendimiento y concentración de las participantes por sobre las demás técnicas. Por otra parte, el ejercicio de la sesión 2, "es- caneo corporal", presentó un sobreesfuerzo fisiológico en los participantes. Asimismo, las estrategias efectuadas entre las sesiones 3-7 sugieren que lograron disminuir la tasa respiratoria, aunque sólo se encontró un efecto estadísticamente significativo en la sesión 4 con la práctica "mindfulness en tu mano", que mostró un efecto. Al principio se plateó medir la temperatura periférica en todas las sesiones. Sin embargo, debido a que cada participante tenía condiciones ambientales distintas por la humedad y la temperatura de su domicilio, se sustituyó la medición psicofisiológica térmica por la tasa respiratoria, como lo sugieren Alcaraz-Mendoza y García-Valencia (2012).

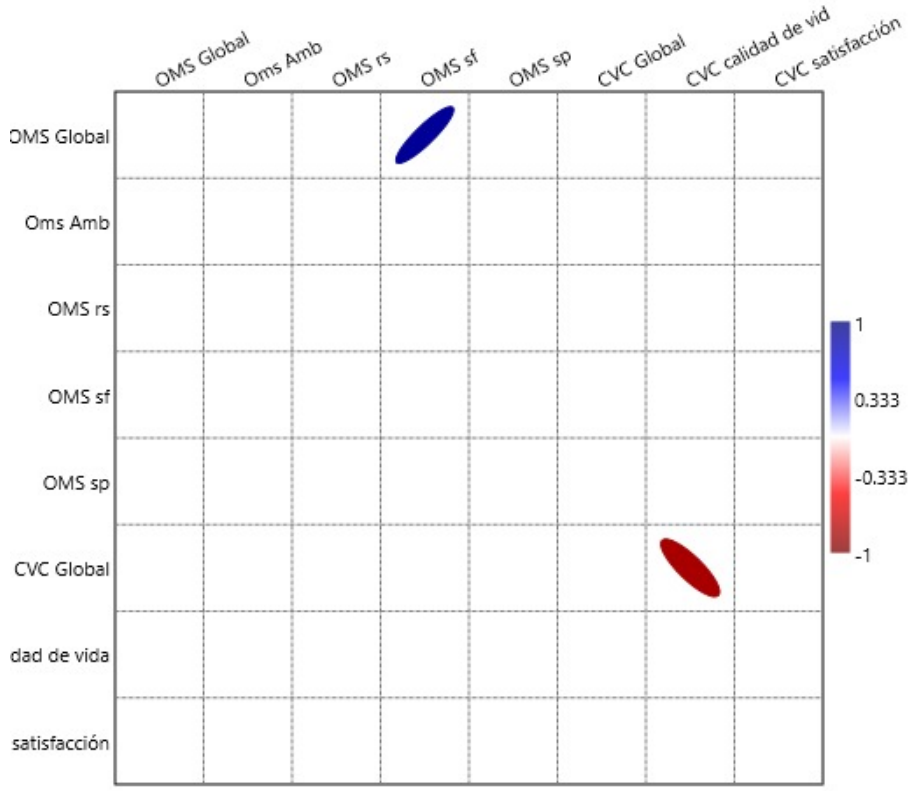

Figura 5.

Correlaciones entre los factores de los instrumentos WHOQOL-BREF y ECVS-FC.

Nota: Las correlaciones directamente proporcionales se muestran en azul, las inversamente proporcionales están en rojo, las resaltadas tienen una diferencia estadísticamente significativa $(\alpha=0.05)$. OMS Amb = factor ambiental, OMS rs $=$ Relaciones sociales, OMS sp = salud psicológica, CVC g = calidad de vida del cuidador global, CVC cV = calidad de vida del cuidador, calidad de vida, CVC s = calidad de vida del cuidador, satisfacción.

Se usaron como base las técnicas de mindfulness propuestas por la página web de la brigada de salud del INCMNSZ; no obstante, no se indica si su aplicación puede ser grupal, a distancia, ni cuantas repeticiones se sugieren para lograr un nivel óptimo de la atención plena; en este sentido, esta investigación alcanza un nivel exploratorio. En la página web de la brigada de salud 
del INCMNSZ no se indica si su aplicación puede ser grupal, a distancia, ni cuantas repeticiones se sugieren para lograr un nivel óptimo de la atención.

En revisiones sistemáticas, hechas en la práctica de mindfulness en cuidadores informales de mayores crónicos dependientes (Verea y Borrego, 2019) y personas con demencia (Grandi, Burgueño y Irurtia., 2018), se han reportado reducciones significativas en sintomatología ansiosa y depresiva, así como disminución de estrés crónico, generando mejorías en la salud general. Sin embargo, no llegaron a tener efecto en la calidad de vida percibida.

De acuerdo con Verea y Borrego (2019), la calidad de vida percibida no se ha podido medir de manera clara, al tratarse de un concepto multidimensional con cargas objetivas y subjetivas. En su revisión sistemática, especifican que los instrumentos utilizados para medir la calidad de vida no parecen ser lo suficientemente sensibles. No obstante, no presentan una propuesta alternativa validada, por lo que sería recomendable diseñar un instrumento sensible y específico a los cuidadores.

El entrenamiento en mindfulness en la modalidad a distancia en el contexto de la pandemia por Covid-19 para el acompañamiento psicológico vía telefónica o por videollamada, presentó variabilidad en múltiples factores, como la heterogeneidad en los perfiles psicológicos de los participantes, la disponibilidad de conexión a Internet, las demandas que implican la tarea de un cuidador primario y la privacidad en su casa para recibir la asistencia psicosocial. No obstante, los efectos en la tasa respiratoria asociada al mindfulness mostraron efectos favorables en la mayoría de las sesiones, como lo reportan los estudios en la modalidad presencial (Ruiz-Fernandez et al., 2012).

También es importante destacar que el efecto del entrenamiento en mindfulness en la tasa respiratoria era más notable en relación con la asistencia de los participantes (sesiones 4 y 7), por lo que es importante un estudio confirmatorio de esta relación. Aunque los cambios en la tasa respiratoria reportados durante las sesiones no presentaros diferencias estadísticamente significativas, es prudente pensar que se mantuvieron en un nivel saludable al margen de la muestra (Rodríguez-Molinero et al., 2013).

Similar a estudios con ejercicios de respiración controlada a voluntad (Cea-Ugarte et al., 2004), la temperatura periférica tiende a elevarse con la práctica, que, aunque para este estudio no representó un cambio significativo, si denotó una tendencia al aumento de la temperatura con el ejercicio de "respirar con atención plena". Sin embargo, con el ejercicio de "escaneo corporal" no se presenció el mismo efecto.

Debido a la duración de la sesión y la disponibilidad limitada de los participantes, es posible que el efecto de la técnica de escaneo corporal no haya logrado el efecto psicofisiológico esperado, porque la fatiga de los participantes se les presentó con la técnica después de más de 100 minutos de acompañamiento psicosocial. Además, es prudente mencionar que esta sesión tuvo algunos problemas de conectividad de varios participantes, y esto pudo ser un factor clave en el efecto de la técnica.

En el caso de las evaluaciones psicométricas, algunas cuidadoras presentaron cambios favorables y desfavorables. Sólo el apoyo instrumental que alude a disponibilidad de ayuda doméstica mostró una diferencia significativa entre el inicio de la investigación y el final de ésta; esto podría deberse al apoyo que percibieron las participantes por parte de sus familiares en las distintas dificultades del modo a distancia de la intervención.

La ansiedad y la depresión no mostraron descensos estadísticamente significativos; no obstante, los índices de ambas variables descendieron en la mayoría de los participantes; esto representa una mejora en la percepción subjetiva en su salud emocional.

Respecto a las correlaciones, las relaciones entre las subescalas del instrumento de calidad de vida podrían sugerir una disociación entre calidad de vida personal y la calidad de vida como cuidador informal; es decir, tener una calidad de vida global no garantiza la calidad de vida de cuidador informal. En el índice de apoyo social se mostró una relación directa entre los factores emocional e instrumental. En la mayoría de los participantes se mejoró la comunicación asertiva, el uso y resignificación de la red de apoyo social que tienen en su vida cotidiana. Por otra parte, se reportó una correlación inversa entre el factor afectivo del apoyo social y el índice de ansiedad; es decir, las muestras de amor y afecto constante tienen una relación con el descenso de la ansiedad de los participantes (Barrera-Herrera et al., 2019).

Entre las limitaciones de este estudio destacan los instrumentos diseñados para ser autoaplicables; no obstante, se adaptó este criterio para ser aplicados mediante Ilamadas telefónicas; esto pudo afectar los resultados en el tiempo de aplicación, las distracciones del medio y el entendimiento de los ítems; también es probable que los participantes pueden sentirse menos inhibidos al llenar el autor el reporte mientras que, al dar las respuestas vía telefónica tengan una tendencia a abstenerse a expresar cómo se sienten en realidad o deseabilidad social. Se sugiere adaptar y diseñar instrumentos psicométricos y métodos de intervención para escenarios a distancia en situaciones extraordinarias, como el contexto ocasionado por la pandemia. 


\section{ConcLusiones}

El acompañamiento psicosocial para promover el autocuidado de la salud y los ejercicios de mindfulness disminuyeron de modo parcial el nivel de estrés, por lo que es necesaria mayor continuidad en la práctica, como los ejercicios en casa, así como dar seguimiento a este grupo para valorar los efectos a largo plazo.

El apoyo social instrumental fue el único que mostró mejoría, en buena parte por la asistencia de los familiares y personas cercanas para lograr involucrarse en el acompañamiento psicosocial, proporcionándoles dispositivos e instrucciones para su conexión y actividades de las sesiones.

\section{Referencias}

Alcaraz Mendoza, F., \& García Valencia, A. (2012). Técnicas psicofisiológicas para evaluar el estrés en escenarios clínicos. En S. Galán Cuevas, \& E. J. Camacho Gutiérrez, Estrés y salud. Investigación básica y aplicada (pp. 73-88). México: El Manual Moderno.

Barrera-Herrera, A., Neira-Cofré, M., Raipán-Gómez, P., Riquelme-Lobos, P. \& Escobar, B. (2019). Apoyo social percibido y factores sociodemográficos en relación a los síntomas de ansiedad, depresión, y estrés en universitarios chilenos. Revista de Psicopatología y Psicología clínica, 24, 105-115. doi: 10.5944/rppc.23676

Bayón, M. C., \& Saraví, G. A. (enero-abril de 2019). Desigualdades: Subjetividad, otredad y convivencia social en Latinoamérica. Desacatos, 8-15. http://www.scielo.org. $\mathrm{mx} / \mathrm{pdf} /$ desacatos/n59/2448-5144-desacatos-59-8. pdf

Berman, A. J., \& Snyder, S. (2013). Fundamentos de enfermeria: Conceptos proceso y practica, (9a. ed.), pp 555-560. Pearson

Camacho-Gutiérrez, E., Vega-Michel, C., Alcaraz-Mendoza, F., González, M. Á., Arellano-Gómez, L., Colunga-Rodríguez, C., Cruz-Gómez, D., González-Araiza, R., GuzmánSescosse, M., Hunot-Alexander, C., Maldonado-Saucedo, M., Meneses-Ortega, S., Orozco-Medina, M. G., JavierPérez, F., Bouquet-Lazcano, Pando-Moreno, M., PliegoRayas, A., Sánchez-Loyo, L. M., Vázquez-Colunga, J. C., Viesca-Lobatón, A., et al. (2014). Autocuidado de la salud, pp. 17-28, 45-54, 152-169. México: ITESO.

Camacho, L., Yokebed, G., \& Jiménez, A. (2010). Sobrecarga del cuidador primario de personas con deterioro cognitivo y su relación con el tiempo de cuidado. Enfermería universitaria, 7(4), 35-41. http://www.scielo. org. $\mathrm{mx} /$ scielo.php?script=sci_arttext\&pid=S1665$70632010000400006 \&$ lng $=$ es\&tlng $=$ es

Cardona-A., D., Segura-C., Á. M., Berbesí-F., D. Y., \& Agudelo-M., M. (2013). Prevalencia y factores asociados al síndrome de sobrecarga del cuidador primario de ancianos. Revista Facultad Nacional de Salud Pública, 31(1), 30-39. https:// www.redalyc.org/articulo.oa?id=12026437003
Carranza, M., Vázquez, J. J., Manjarrez, J. O., Pérez, M., Rodríguez, D. \& Ramírez, A. (2020). Intervención psicosocial ante la pandemia por Covid-19 en México. En O. E. Infante Pedreira, \&J. A. Grau Ávalo, La psicología de la salud en el enfrentamiento a la Covid-19 en América Latina (pp. 206249). Santiago de Chile: CEDEM. https://alapsa.net/wpcontent/uploads/2021/02/alapsa2021a.pdf

Cea-Ugarte, J. I., Cabo-González, O., González-Pinto, A., \& BrazalRaposo, J. J. (2004). Efectos beneficiosos de la respiración manejada a voluntad sobre la frecuencia cardiaca y temperatura periférica. Psiquis: Revista de psiquiatría, psicología médica y psicosomática, 25(2), 17-26. https:// hipnosis.org/documentos/escuela/articulos/Efectosbeneficiosos-respiraci\%C3\%B3n-sobre-frecuenciacard\%C3\%ADaca-temperatura-perif\%C3\%A9rica-JIgnacio-Cea.pdf

Cisneros-Huerta, A. (2007). Validez y confiabilidad del cuestionario general de salud SF-12 en derechohabientes de una unidad de primer nivel del Instituto Mexicano del Seguro Social. México: Universidad Veracruzana. http:// cdigital.uv.mx/handle/123456789/32287

Diez-Quevedo, C., Rangil, T., Sanchez-Planell, L., Kroenke, K., \& Spitzer, R. (2001). Validation and Utility of the Patient Health Questionnaire in Diagnosing Mental Disorders in 1003 General Hospital Spanish Inpatients. Psychosomatic Medicine, 63(4), 679-686. https://doi. org/10.1097/00006842-200107000-00021

Expósito, M., Martín, M., \& Oviedo, H. (2019). La ESCV-FC para evaluar calidad de vida en cuidadores primarios en el Caribe colombiano. Index de Enfermería, 27(3), 152155. https://ciberindex.com/index.php/ie/article/view/ e11804

Expósito Concepción, M. Y., Martín Carbonell, M., \& Oviedo Córdoba, H. (2018). La ESCV-FC para evaluar Calidad de Vida en cuidadores primarios en el Caribe colombiano. Index De Enfermería, 27(3), 152-155. Recuperado a partir de http://ciberindex.com/c/ie/e11804

Grandi, F., Burgueño, L., \& Irurtia, M. J. (2018). Eficacia del programa de reducción del estrés basado en mindfulness para cuidadores familiares de personas con demencia. Revisión sistemática de estudios clínicos aleatorizados. Revista Española de Geriatría y Gerontología. https:// www.sciencedirect.com/science/article/abs/pii/ S0211139X18306735?via\%3Dihub

Hernández-Cantú, E. I., Reyes-Silva, A. K., Villegas-García, V. E., \& Pérez-Camacho, J. (2017). Ansiedad, depresión y sobrecarga en cuidadores primarios de pacientes internados en un hospital general de zona en Nuevo León, México. Rev Enferm Inst Mex Seguro Soc., 25(3), 213-220. https://www.medigraphic.com/pdfs/enfermeriaimss/ eim-2017/eim173h.pdf

Hernández Sampieri, R., Fernández Collado, C., \& Baptista Lucio, M. D. (2014). Metodología de la investigación, 6a. ed. México: Mc Graw-Hill. http://observatorio.epacartagena. gov.co/wp-content/uploads/2017/08/metodologia-dela-investigacion-sexta-edicion.compressed.pdf

Instituto Nacional de Ciencias Médicas y Nutrición Salvador Zubirán (INCMNSZ) (2020). INCMNSZ. Brigada de Salud Mental. http://www.incmnsz.mx/opencms/contenido/ 
BrigadaSaludMental/index.html

Ioannou, S., Gallese, V., \& Merla, A. (2014). Thermal infrared imaging in psychophysiology: Potentialities and limits. Psychophysiology, 51, 951-963. https://doi. org/10.1111/psyp.12243

Islas, L., Ramos, B., Aguilar M., \& García, M. (2006). Perfil psicosocial del cuidador primario informal del paciente con EPOC. Revista del Instituto Nacional de Enfermedades Respiratorias, 19(4), 266-271. http://www.scielo.org.mx/ pdf/iner/v19n4/v19n4a6.pdf

Kabat-Zinn, J. (1990). Full catastrophe living. How to cope with stress, pain and illness using mindfulness meditation. NY: Piadkus.

López-Huerta, J. A., González-Romo, R. A., \& Tejada-Tayabas, J. M. (2017). Propiedades psicométricas de la versión en español de la escala de calidad de vida WHO QoL BREF en una muestra de adultos mexicanos. Revista Iberoamericana de Diagnóstico y Evaluación, 44(2), 105115. https://doi.org/10.21865/RIDEP44.2.09

Löwe, B., Wahl, I., Rose, M., Spitzer, C., Glaesmer, H., Wingenfeld, K., Schneider, A., \& Brähler, E. (2010). A 4-item measure of depression and anxiety: Validation and standardization of the Patient Health Questionnaire-4 (PHQ-4) in the general population. Journal of Affective Disorders, 86-95. https:// doi.org/10.1016/j.jad.2009.06.019

Organización Mundial de la Salud (OMS) (1996). WHOQOL-BREF Introduction, administration, scoring and generic version of the assessment. Geneva: OMS. https://www.who.int/ mental_health/media/en/76.pdf

Organización Mundial de la Salud (OMS) (1998). Development of the World Health Organization WHOQOL-BREF Quality of Life Assessment. Psychologycal Medicine, 28, 551-558. https://doi:10.1017/S003329179800666

Ortega-Ruiz, R. (2006). La convivencia: un modelo de prevención de la violencia. En A. Moreno-González, \& M. P. SolerVillalobos, La convivencia en las aulas: Problemas y soluciones (pp. 29-48). Esáña: Ministerio de Educación y Ciencia.

Prins, A., Ouimette, P., Kimerling, R., Cameron, R. P., Hugelshofer, D. S., Shaw-Hegwer, J., Thrailkil, A., Gusman, F. D., \& Sheikh, J. I. (2003). The primary care PTSD screen (PC-PTSD): development and operating characteristics. Librapharm Limited, 9(1), 9-14. https://www.ptsd.va.gov/PTSD/ professional/articles/article-pdf/id26676.pdf

Puerto-Pedraza, H. M., \& Carrillo-González, G. M. (2015). Calidad de vida y soporte social en los cuidadores familiares de personas en tratamiento contra el cáncer. Rev Univ Ind Santander Salud, 47(2), 125-136. https://www.redalyc. org/pdf/3438/343839278004.pdf

Rodríguez-Medina, D., Domínguez, B., Leija, G, Ortiz, M, \& Carreño, C. (2018). Efectos psicofisiológicos de la respiración diafragmática y la estimulación térmica sobre la actividad autonómica del estrés agudo. Acta de Investigación Psicológica, 8(2), 101-113. https://doi. org/10.22201/fpsi.20074719e.2018.2.09

Rodríguez, D., Vázquez Ortega, J. J., \& Alonso Hernández, G. (2020). Evaluación e intervención psicofisiológica térmica de sesión única de estrés social en estudiantes de Psicología. Revista Digital Internacional de Psicología y Ciencia Social, 6(2), 340-354. https://cuved.unam.mx/ revistas/index.php/rdpcs/article/view/227/629

Rodríguez-Medina, D. A., \& Hernández-Pozo, M. D. (2021). Termorregulación afectiva: Funcionamiento biopsicosocial de la respuesta de estrés social en universitarios postsismo del 19s. Acta de Investigación Psicológica, 11(1), $73-$ 83. https://www.revista-psicologia.unam.mx/revista_ aip/index.php/aip/article/view/375/332

Rodríguez-Molinero, A., Narvaiza, L., Ruiz, J., \& Gálvez-Barrón, C. (2013). Normal Respiratory Rate and Peripheral Blood Oxygen Saturation in Elderly Population. Journal of the American Geriatric Society. https://doi.org/10.1111/ jgs. 12580

Ruiz-Fernández, M. A., Díaz-García, M. I., \& Villalobos-Crespo, A. (2012). Manual de técnicas de intervención Cognitivo Conductuales. UNED.

Sherbourne, C. D., \& Stewart, A. L. (1991). The MOS social support survey. Social Science \& Medicine, 32(6), 705-714. https://doi.org/10.1016/0277-9536(91)90150-b

Verea, M., \& Borrego, R. (2019). Educación sanitaria en mindfulness a los cuidadores de mayores crónicos dependientes. Medicina Naturista, 78-90. https://dialnet. unirioja.es/servlet/articulo?codigo $=6985228$ 


\section{Meta-Análisis del Artículo}


Artículo Empírico | Acompañamiento psicosocial en cuidadores primarios... | Vicente-Ramírez, et al.

\section{Dimensión Cuantitativa}

\section{Perfil de Evaluación entre pares}
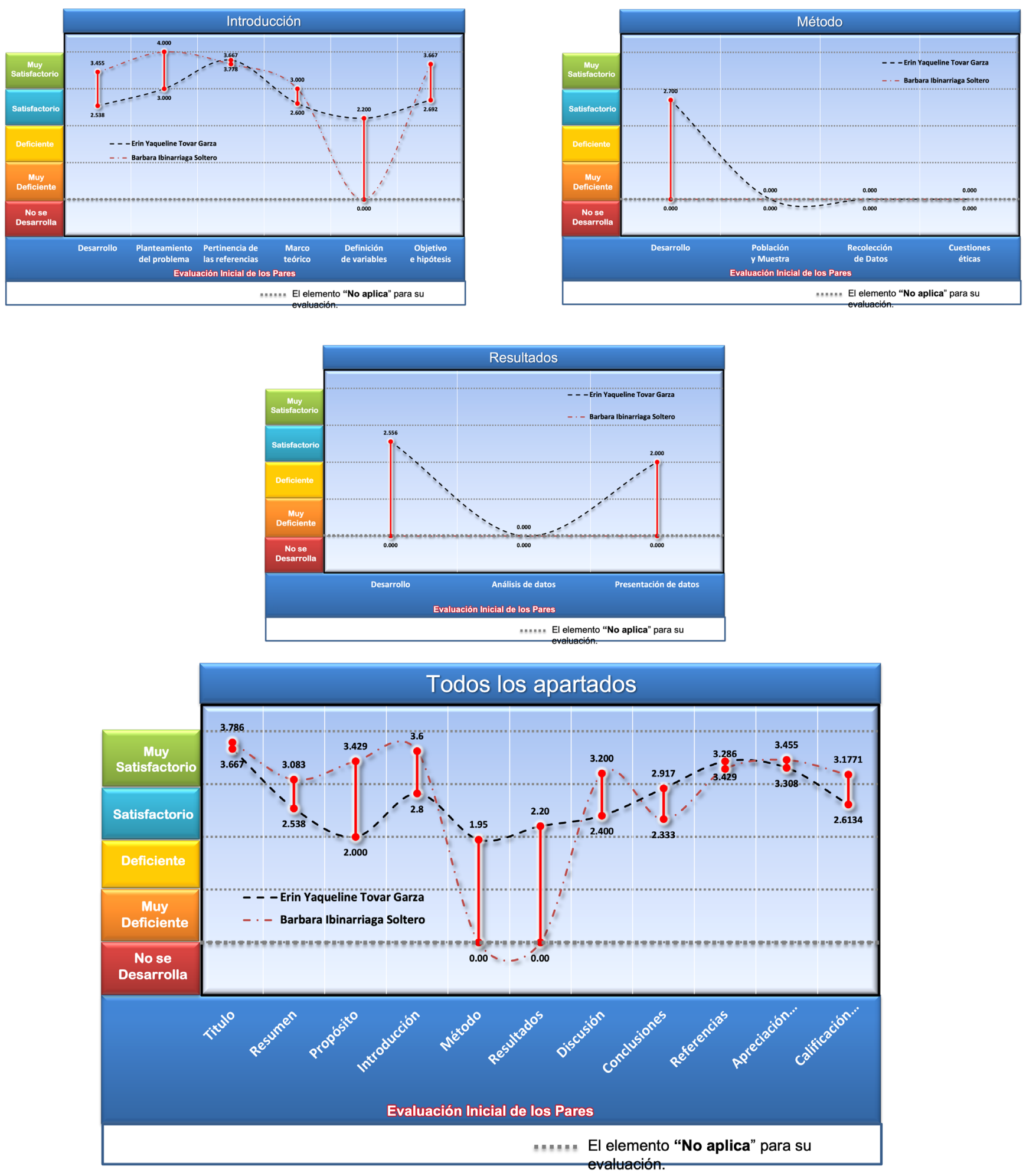


\section{Índice de Concordancia}

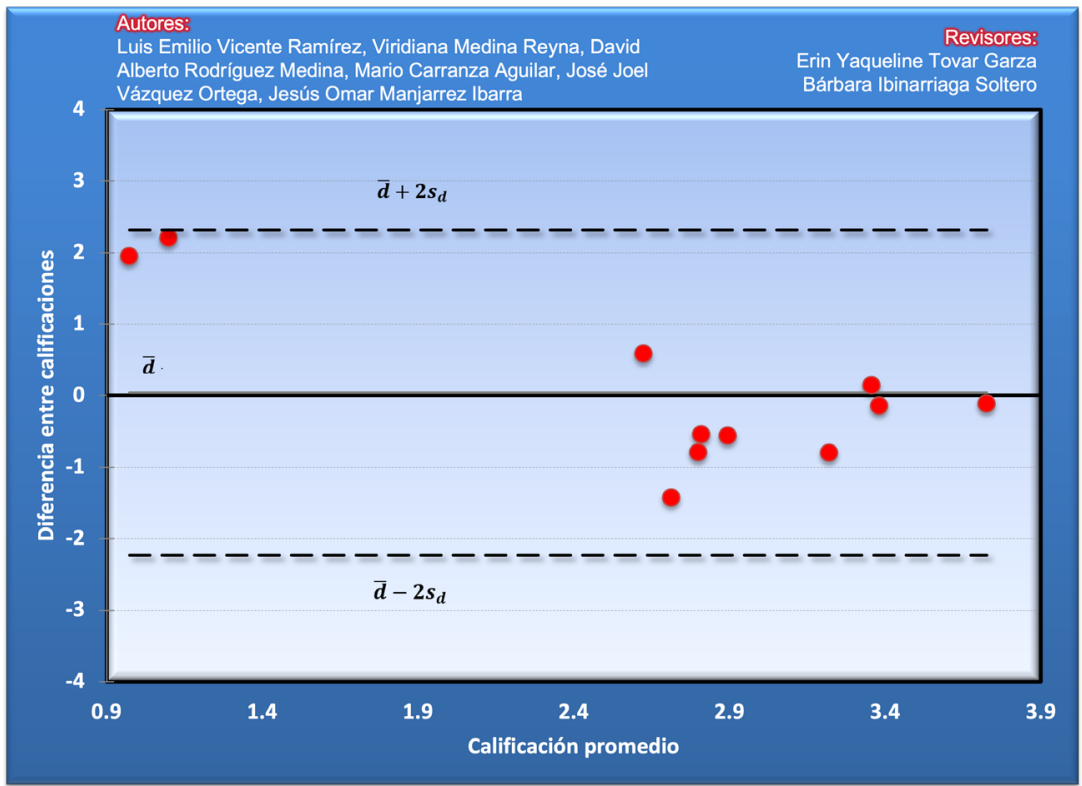

Índice de Acuerdo

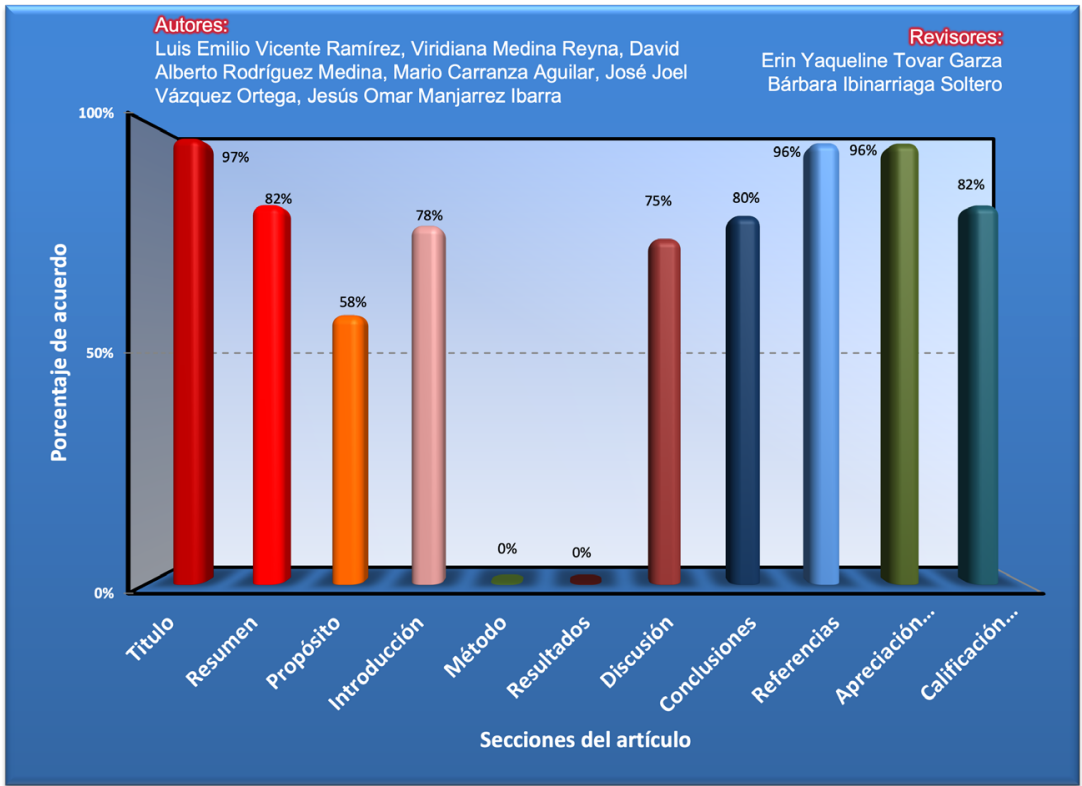




\begin{tabular}{|c|}
\hline \multicolumn{1}{|c|}{ Revisor 1 } \\
\hline \multicolumn{1}{|c|}{ Erin Yaqueline Tovar Garza } \\
\hline \multicolumn{1}{|c|}{ Títul } \\
Tu título y tu objetivo manejan variables distintas en el ob- \\
jetivo hablas de practicas de promoción y prevención de \\
la salud y en el titulo de acompañamiento psicosocial, te \\
recomiendo homogeneizar los términos.
\end{tabular}

Se proporciona un correo electrónico, no el de todos los autores. De igual modo, únicamente se explica la contribución en la realización del trabajo de 3 autores, faltando la descripción de los otros 3 autores.

\section{Resumen}

Se mencionan técnicas como el uso de la escucha activa y el intercambio de experiencias psicosociales y variables como la salud física y emocional que no se describen en ninguna parte del manuscrito Falta poner los coeficientes de correlación junto con la descripción de los resultados. El resumen es de 228 palabras, cuando piden 150 La palabras clave asertividad y autocuidado no se reflejan en ninguna parte del manuscrito, te recomiendo usar las variables que presentas en tu título aparecen como palabras clave.
El resumen puede ser más claro explicando mejor el número de sesiones del programa (8 en total) y el tipo de mediciones utilizadas. Aunque esto se desarrolla en los resultados, al leer el resumen es confuso. En la evaluación cuantitativa se señala que el resumen debe contener un máximo de 150 palabras, pero este número se rebasa (más de 200 palabras). Considero que la información incluida es adecuada desde mi punto de vista, pero quizá se deba considerar el requisito de la longitud. Por otro lado, he propuesto algunas ideas para que la versión en inglés del resumen sea más precisa. Finalmente, considero que la palabra mindfulness debe de estar en el grupo de las palabras clave.

\section{Próposito del Estudio}

Tu objetivo no concuerda con tu título, falta complementar que es adaptad para la distancia. No todos los términos del propósito no se mencionan en la introducción
Aunque el propósito está redactado de forma clara, no encontré dicha redacción en forma de pregunta(s) de investigación. Quizá formular dichas pregunta(s) de investigación ayudaría precisamente a presentar los resultados relativos a las preguntas. Un elemento que sería importante a desarrollar más, es cómo se define el aspecto de acompañamiento, pues aunque se da una descripción del temario de las sesiones del programa de mindfulness, los autores podrían enfatizar más cómo es que dicha intervención está vinculada al proceso de acompañamiento psicosocial en la discusión del artículo. 


\begin{tabular}{|c|c|}
\hline Revisor 1 & Revisor 2 \\
\hline \multicolumn{2}{|c|}{ Introducción } \\
\hline $\begin{array}{l}\text { No todas tus variables se explican en la introducción y } \\
\text { el nombre de estas variables cambian constantemente. } \\
\text { El hilo conductor de la introducción al propósito no es } \\
\text { claro Falta describir claramente como operacionalizas } \\
\text { cada variable }\end{array}$ & $\begin{array}{l}\text { Podría justificar por qué utilizar la práctica de mind- } \\
\text { fulness y no otro tipo de intervención. La revisión de } \\
\text { literatura se beneficiaría de revisar más fuentes interna- } \\
\text { cionales y nacionales sobre las intervenciones de mind- } \\
\text { fulness, y de otras intervenciones realizadas a distancia } \\
\text { debido a Covid-19 (si no hubiese datos disponibles so- } \\
\text { bre el último punto, los autores pueden decir que ésta } \\
\text { es parte de la aportación). Es necesario identificar las } \\
\text { variables extrañas y cómo estas fueron controladas (en } \\
\text { el texto se habla de una muestra heterogénea, pero no } \\
\text { se especifica la razón). Aunque las variables se definen } \\
\text { de acuerdo a los instrumentos de medición utilizados, } \\
\text { no existe como tal la redacción de las hipótesis en el } \\
\text { artículo. Por esta razón, he seleccionado }{ }^{*} \text { no aplica* en } \\
\text { el cuestionario cuantitativo. }\end{array}$ \\
\hline \multicolumn{2}{|c|}{ Método } \\
\hline $\begin{array}{l}\text { No queda claro porque utilizas cada instrumento, a } \\
\text { variable hace referencia Falta desarrollar como fue tu } \\
\text { muestreo }\end{array}$ & $\begin{array}{l}\text { La sección de método no describe el procedimiento para } \\
\text { obtener el consentimiento informado de los participan- } \\
\text { tes. Tampoco se describen todas las características socio- } \\
\text { demográficas de la muestra ni el porcentaje de asistencia } \\
\text { de los participantes a las sesiones (variable importante } \\
\text { que los autores discuten en relación a los resultados). Se- } \\
\text { ría pertinente discutir el impacto del tamaño de la mues- } \\
\text { tra en la discusión de los resultados, así como las limita- } \\
\text { ciones metodológicas debido a que la intervención fue } \\
\text { realizada a distancia y por tanto la cautela que se debe de } \\
\text { tomar en el análisis de las mediciones. }\end{array}$ \\
\hline \multicolumn{2}{|c|}{ Resultados } \\
\hline $\begin{array}{l}\text { Parte del procedimiento se encuentra en el apartado de } \\
\text { resultados. La figuras utilizadas son muy confusas y no } \\
\text { muestran los coeficientes de correlación exactos. Los } \\
\text { subtitulos no tiene relación con las variables estudiadas } \\
\text { No aparecen los valores estadísticos que se encontra- } \\
\text { ron, solo su interpretación. }\end{array}$ & $\begin{array}{l}\text { El orden de la presentación de los resultados difiere de la } \\
\text { secuencia en la que se presentaron los instrumentos de } \\
\text { medición. Ayudaría tener consistencia entre estas dos sec- } \\
\text { ciones. Debido a que existieron algunas complicaciones } \\
\text { con una de las mediciones fisiológicas, los autores decidie- } \\
\text { ron presentar estas medidas primero. Describir estas limi- } \\
\text { taciones es importante. Sin embargo, este criterio difiere } \\
\text { con las preguntas cuantitativas de esta sección. }\end{array}$ \\
\hline
\end{tabular}




\begin{tabular}{|c|c|}
\hline Revisor 1 & Revisor 2 \\
\hline \multicolumn{2}{|c|}{ Discusión } \\
\hline $\begin{array}{l}\text { La redacción es repetitiva en el primer y segundo pá- } \\
\text { rrafo. Hace falta mostrar los resultado o conclusiones } \\
\text { que se encontraron en los estudios que se usan para } \\
\text { la discusión. Agregar estudios que sustenten las expli- } \\
\text { caciones alternativas que se dan de los resultados. Se } \\
\text { sugiere sustentar las interpretaciones en estudios o teo- } \\
\text { rías previas. }\end{array}$ & $\begin{array}{l}\text { Considero que es necesario expandir en las hipótesis al- } \\
\text { ternativas que explican los diferentes resultados del estu- } \\
\text { dio, así como expandir en detalles que ayudan a enten- } \\
\text { der el impacto de la intervención (por ejemplo, el tipo de } \\
\text { apoyo que los participantes recibieron por parte de sus } \\
\text { familiares). También es necesario ampliar las discusión } \\
\text { sobre la inclusión de métodos cualitativos para investigar } \\
\text { la percepción de calidad de vida de los individuos, dadas } \\
\text { las posibles diferencias entre calidad de vida global y la } \\
\text { calidad del cuidador/a. }\end{array}$ \\
\hline \multicolumn{2}{|c|}{ Conclusiones } \\
\hline $\begin{array}{l}\text { Algunas de las frases mencionadas van en el apartado de } \\
\text { resultados o discusión. }\end{array}$ & $\begin{array}{l}\text { Las conclusiones son bastante breves y podrían expan- } \\
\text { dirse para incluir la significancia social de los resulta- } \\
\text { dos, por ejemplo en el contexto del Sistema Público de } \\
\text { Cuidados de la Ciudad de México; así como proponer } \\
\text { investigaciones futuras. De acuerdo a la evaluación } \\
\text { cuantitativa y los criterios mencionados ahí, las con- } \\
\text { clusiones del estudio deben de relacionar los resultados } \\
\text { con los objetivos; los autores realizaron esto en la sec- } \\
\text { ción de discusión. }\end{array}$ \\
\hline \multicolumn{2}{|c|}{ Referencias } \\
\hline $\begin{array}{l}\text { Solo se presentan } 23 \text { referencias, tratar de aumentar a al } \\
\text { menos } 30 .\end{array}$ & $\begin{array}{l}\text { En el manuscrito mencioné los lugares en donde es } \\
\text { necesario incorporar más referencias. También es ne- } \\
\text { cesario revisar el formato para que sea consistente al } \\
\text { citar los autores con doble apellido y el uso de guión. } \\
\text { Las referencias señaladas debajo no están citadas en el } \\
\text { texto, por lo que es necesario hacer los cambios nece- } \\
\text { sarios: Akintui Villalobos, Y. D., \& López Araujo, C. } \\
\text { A. (2014). Asociación entre calidad de vida y nivel de } \\
\text { sobrecarga del cuidador primario de paciente con en- } \\
\text { fermedad renal crónica. UNIVERSIDAD CATÓLICA } \\
\text { SANTO TORIBIO DE MOGROVEJO, 1-48. http://hdl. } \\
\text { handle.net/20.500.12423/305 Villalobos, A., Díaz, M. } \\
\text { I., \& Ruis, M. Á. (2012). Mindfulness. En M. Á. Ruíz } \\
\text { Fernández, M. I. Díaz García, \& A. Villalobos Crespo, } \\
\text { Manual de técnicas de intervención cognitivo conduc- } \\
\text { tuales (págs. 467-512). México: Desclée de Brouwer. }\end{array}$ \\
\hline
\end{tabular}




\section{Historia del Proceso EDITORIAL}

